\title{
STATE SPACE FORMULAS FOR A SUBOPTIMAL RATIONAL LEECH PROBLEM I: MAXIMUM ENTROPY SOLUTION
}

\author{
A.E. FRAZHO, S. TER HORST, AND M.A. KAASHOEK
}

\begin{abstract}
For the strictly positive case (the suboptimal case) the maximum entropy solution $X$ to the Leech problem $G(z) X(z)=K(z)$ and $\|X\|_{\infty}=$ $\sup _{|z| \leq 1}\|X(z)\| \leq 1$, with $G$ and $K$ stable rational matrix functions, is proved to be a stable rational matrix function. An explicit state space realization for $X$ is given, and $\|X\|_{\infty}$ turns out to be strictly less than one. The matrices involved in this realization are computed from the matrices appearing in a state space realization of the data functions $G$ and $K$. A formula for the entropy of $X$ is also given.
\end{abstract}

\section{INTRODUCTION}

Let $G$ and $K$ be matrix-valued $H^{\infty}$ functions on the open unit disc $\mathbb{D}$ of sizes $m \times p$ and $m \times q$, respectively, and let $T_{G}$ and $T_{K}$ denote the corresponding block lower triangular Toeplitz operators,

$$
T_{G}: \ell_{+}^{2}\left(\mathbb{C}^{p}\right) \rightarrow \ell_{+}^{2}\left(\mathbb{C}^{m}\right), \quad T_{K}: \ell_{+}^{2}\left(\mathbb{C}^{q}\right) \rightarrow \ell_{+}^{2}\left(\mathbb{C}^{m}\right) .
$$

A $p \times q$ matrix-valued $H^{\infty}$ function $X$ is called a solution to the Leech problem associated with $G$ and $K$ whenever

$$
G(z) X(z)=K(z) \quad(z \in \mathbb{D}) \quad \text { and } \quad\|X\|_{\infty}=\sup _{z \in \mathbb{D}}\|X(z)\| \leq 1 .
$$

The Leech problem is an example of a metric constrained interpolation problem, the first part of (1.1) is the interpolation condition, and the second part is the metric constraint. In a note dating from 1971/1972, only published recently [18, see also [17, Leech proved that the problem is solvable if and only if the operator $T_{G} T_{G}^{*}-T_{K} T_{K}^{*}$ is nonnegative. Later the Leech theorem was derived as a corollary of more general results; see, e.g., [19, page 107], 8, Section VIII.6]), and [2, Section 4.7].

Now assume in addition that $G$ and $K$ are rational. In other words, assume that $G$ and $K$ are stable rational matrix functions. In that case, if the Leech problem associated with $G$ and $K$ is solvable, one expects the problem to have a stable rational matrix solution as well. However, a priori this is not clear, and the existence of rational solutions was proved only recently in [20] by reducing the problem to polynomials, in [16] by adapting the lurking isometry method used in [3], and in [11] by using a state space approach.

In the present paper $G$ and $K$ are also stable rational matrix functions. We assume additionally that the operator $T_{G} T_{G}^{*}-T_{K} T_{K}^{*}$ is strictly positive. It is then known from commutant lifting theory that the Leech problem has a unique

1991 Mathematics Subject Classification. Primary 47A57; Secondary 47A68, 93B15, 47A56.

Key words and phrases. Leech problem, stable rational matrix functions, commutant lifting theorem, state space representations, algebraic Riccati equation. 
maximum entropy solution, that is, the (unique) solution $X$ to the Leech problem associated with $G$ and $K$ for which the quantity

$$
\mathcal{E}(X)=\frac{1}{2 \pi} \int_{0}^{2 \pi} \ln \operatorname{det}\left[I_{q}-X\left(e^{\imath \omega}\right)^{*} X\left(e^{\imath \omega}\right)\right] d \omega
$$

is maximal. In this paper we show that this maximum entropy solution is a stable rational matrix function, we derive an explicit formula for this solution and a formula for its entropy $\mathcal{E}(X)$; see Theorem 1.2 below. When $T_{G} T_{G}^{*}-T_{K} T_{K}^{*}$ is only non-negative, the maximum entropy solution still exists but the problem whether or not it is rational remains open.

To prove the above mentioned results, we use the fact, well-known from mathematical systems theory (see, e.g., Chapter 1 of [7] or Chapter 4 in [4]), that rational matrix functions admit state space realizations. For our $G$ and $K$ this means that the matrix function $\left[\begin{array}{ll}G & K\end{array}\right]$ admits a representation of the following form:

$$
[G(z) \quad K(z)]=\left[\begin{array}{ll}
D_{1} & D_{2}
\end{array}\right]+z C\left(I_{n}-z A\right)^{-1}\left[\begin{array}{ll}
B_{1} & B_{2}
\end{array}\right] .
$$

Here $I_{n}$ is the $n \times n$ identity matrix, $A$ is an $n \times n$ matrix, and $B_{1}, B_{2}, C, D_{1}$ and $D_{2}$ are matrices of appropriate sizes. Moreover, since $G$ and $K$ are stable rational matrix functions, $G$ and $K$ have no pole in the closed unit disc, and therefore we may assume that matrix $A$ is stable, that is, $A$ has all its eigenvalues in the open unit disc. The realization (1.3) is called minimal if there exists no realization of $\left[\begin{array}{ll}G & K\end{array}\right]$ as in (1.3) with 'state matrix' $A$ of smaller size than the one in the given realization. In that case the order $n$ of $A$ is called the McMillan degree of $\left[\begin{array}{ll}G & K\end{array}\right]$. If the realization (1.3) is minimal, then the matrix $A$ is automatically stable and the observability operator $W_{o b s}$, which is defined by

$$
W_{\text {obs }}=\left[\begin{array}{c}
C \\
C A \\
C A^{2} \\
\vdots
\end{array}\right]: \mathbb{C}^{n} \rightarrow \ell_{+}^{2}\left(\mathbb{C}^{m}\right),
$$

is one-to-one. In the sequel we do not require the realization (1.3) to be minimal but we shall always assume that $A$ is stable and $W_{o b s}$ is one-to-one. In that case we refer to (1.3) as an observable stable realization.

As a first step towards our main result we first derive, in Theorem 1.1 below, a necessary and sufficient condition for $T_{G} T_{G}^{*}-T_{K} T_{K}^{*}$ to be strictly positive in terms of the matrices in (1.3) and related matrices. To do this we need the rational $m \times m$ matrix function

$$
R(z)=G(z) G^{*}(z)-K(z) K^{*}(z) .
$$

Here $G^{*}(z)=G\left(\bar{z}^{-1}\right)^{*}$ and $K^{*}(z)=K\left(\bar{z}^{-1}\right)^{*}$. Note that $R$ has no pole on the unit circle $\mathbb{T}$. By $T_{R}$ we denote the Toeplitz operator defined by $R$. Using the realization (1.3) one shows (see [11, Lemma 3.1]) that $R$ admits the following state space representation:

$$
R(z)=z C(I-z A)^{-1} \Gamma+R_{0}+\Gamma^{*}\left(z I-A^{*}\right)^{-1} C^{*} .
$$

Here $R_{0}$ and $\Gamma$ are matrices of sizes $m \times m$ and $n \times m$, respectively, defined by

$$
\begin{aligned}
R_{0} & =D_{1} D_{1}^{*}-D_{2} D_{2}^{*}+C\left(P_{1}-P_{2}\right) C^{*}, \\
\Gamma & =B_{1} D_{1}^{*}-B_{2} D_{2}^{*}+A\left(P_{1}-P_{2}\right) C^{*},
\end{aligned}
$$


and $P_{1}$ and $P_{2}$ are the unique $n \times n$ matrix solutions of the symmetric Stein equations:

$$
P_{1}-A P_{1} A^{*}=B_{1} B_{1}^{*} \quad \text { and } \quad P_{2}-A P_{2} A^{*}=B_{2} B_{2}^{*} .
$$

Since $A$ is stable, the above equations are solvable and the solutions are unique. Finally, we associate with $R$ the algebraic Riccati equation:

$$
Q=A^{*} Q A+\left(C-\Gamma^{*} Q A\right)^{*}\left(R_{0}-\Gamma^{*} Q \Gamma\right)^{-1}\left(C-\Gamma^{*} Q A\right)
$$

We are now ready to state our main results.

Theorem 1.1. Let $G$ and $K$ be stable rational matrix functions, and assume that $\left[\begin{array}{ll}G & K\end{array}\right]$ is given by the observable stable realization (1.3). Then the operator $T_{G} T_{G}^{*}-T_{K} T_{K}^{*}$ is strictly positive if and only if the following two conditions hold.

(i) There exists a strictly positive $n \times n$ matrix $Q$ such that

(a) $R_{0}-\Gamma^{*} Q \Gamma$ is strictly positive,

(b) $Q$ satisfies the Riccati equation (1.10),

(c) the matrix $A_{0}=A-\Gamma\left(R_{0}-\Gamma^{*} Q \Gamma\right)^{-1}\left(C-\Gamma^{*} Q A\right)$ is stable.

(ii) The operator $Q^{-1}+P_{2}-P_{1}$ is strictly positive.

In this case, the Toeplitz operator $T_{R}$ is strictly positive and the inverse of the operator $T_{G} T_{G}^{*}-T_{K} T_{K}^{*}$ is given by

$$
\begin{gathered}
\left(T_{G} T_{G}^{*}-T_{K} T_{K}^{*}\right)^{-1}=T_{R}^{-1}+T_{R}^{-1} W_{o b s} \Omega W_{o b s}^{*} T_{R}^{-1}, \text { where } \\
\Omega=\left(P_{1}-P_{2}\right)\left(Q^{-1}+P_{2}-P_{1}\right)^{-1} Q^{-1}=\left(P_{1}-P_{2}\right)\left(I+Q\left(P_{2}-P_{1}\right)\right)^{-1} .
\end{gathered}
$$

The second main result shows that the maximum entropy solution is rational and provides a state space realization for this solution.

Theorem 1.2. Let $G$ and $K$ be stable rational matrix functions, and assume that $\left[\begin{array}{ll}G & K\end{array}\right]$ is given by the observable stable realization (1.3). Furthermore, assume that $T_{G} T_{G}^{*}-T_{K} T_{K}^{*}$ is strictly positive, or equivalently, that items $(i)$ and $(i i)$ of Theorem 1.1 hold. Then the maximal entropy solution $X$ to the Leech problem for $G$ and $K$ is a stable rational matrix function which is given by the following state space realization:

$$
X(z)=D_{U} D_{V}^{-1}+z\left(C_{1}-D_{U} D_{V}^{-1} C_{2}\right)\left(I-z A^{\times}\right)^{-1} B_{0} D_{V}^{-1} .
$$

Here, using the matrices appearing in Theorem 1.1, the matrices in (1.12) are defined by

$$
\begin{aligned}
\Delta & =R_{0}-\Gamma^{*} Q \Gamma, \quad C_{0}=\Delta^{-1}\left(C-\Gamma^{*} Q A\right), \quad A_{0}=A-\Gamma C_{0} ; \\
C_{j} & =D_{j}^{*} C_{0}+B_{j}^{*} Q A_{0}, \quad(j=1,2) ; \\
D_{0} & =\Delta^{-1}\left(D_{2}-\Gamma^{*} Q B_{2}\right)+C_{0} \Omega C_{2}^{*} ; \\
B_{0} & =B_{2}-\Gamma \Delta^{-1}\left(D_{2}-\Gamma^{*} Q B_{2}\right)+A_{0} \Omega C_{2}^{*} ; \\
D_{U} & =D_{1}^{*} D_{0}+B_{1}^{*} Q B_{0}, \quad D_{V}=I_{q}+D_{2}^{*} D_{0}+B_{2}^{*} Q B_{0} ; \\
A^{\times} & =A_{0}-B_{0} D_{V}^{-1} C_{2} .
\end{aligned}
$$

Moreover, the state matrix $A^{\times}$is stable, the matrix $D_{V}$ is strictly positive, and the entropy of $X$ is given by

$$
\mathcal{E}(X)=-\ln \operatorname{det}\left[D_{V}\right]
$$


Finally, $\|X\|_{\infty}=\sup _{|z| \leq 1}\|X(z)\|$ is strictly less than one, and the McMillan degree of $X$ is less than or equal to the McMillan degree of $\left[\begin{array}{ll}G & K\end{array}\right]$.

A description of all solutions to the Leech problem (1.1) for the case when $G$ and $K$ are rational will be the topic of a future publication.

Remark 1.3. An $n \times n$ matrix $Q$ is said to be a stabilizing solution of the algebraic Riccati equation (1.10) whenever $Q$ satisfies the three conditions (a), (b) and (c) in item (i) of Theorem 1.1. In this case $Q$ is uniquely determined, cf., formula (1.18) below. Moreover, the existence of a stabilizing solution of (1.10) is equivalent to the Toeplitz operator $T_{R}$ being strictly positive. In that case, the stabilizing matrix $Q$ is given by

$$
Q=W_{o b s}^{*} T_{R}^{-1} W_{o b s} .
$$

See, e.g., 10, Section 10.3], [5, Section 14.7], and [12, Theorem 1.1] for a nonsymmetric version. Also note that there exist several efficient numerical algorithms to compute a stabilizing solution, cf., [1].

The special case of Leech's theorem with $q=m$ and $K$ identically equal to the $m \times m$ identity matrix $I_{m}$ is part of the corona theorem, which is due to Carlson [6], for $m=1$, and Fuhrmann [15] for arbitrary $m$. The least squares solution of the corona version of the equation can be found in 13 and a description of all solutions without any norm constraint in 14. For an engineering perspective on corona and Leech type problems and related applications in signal processing we refer to [22, 21] and the references therein.

The paper consists of five sections including the present introduction. In Section 2 we recall the basic results from commutant lifting theory used in the present paper, and we specify these results for the Leech problem. In Section 3 we assume that $T_{G} T_{G}^{*}-T_{K} T_{K}^{*}$ is strictly positive and, using the commutant lifting results, we derive an infinite dimensional state space realization for the maximum entropy solution. These two sections do not require $G$ and $K$ to be rational; the next two sections do. In Section 4 we further clarify the role of the Toeplitz operator $T_{R}$ with $R$ being given by (1.5) and prove Theorem 1.1. The proof of Theorem 1.2 is given in the final section. At the end of the final section we present a direct proof of the fact that the function $X$ given by (1.12) satisfies the first identity in (1.1) (see Remark 5.3).

Some terminology and notation. For any positive integer $k$ we write $E_{k}$ for the canonical embedding of $\mathbb{C}^{k}$ onto the first coordinate space of $\ell_{+}^{2}\left(\mathbb{C}^{k}\right)$, that is,

$$
E_{k}=\left[\begin{array}{lllll}
I_{k} & 0 & 0 & 0 & \cdots
\end{array}\right]^{\top}: \mathbb{C}^{k} \rightarrow \ell_{+}^{2}\left(\mathbb{C}^{k}\right) .
$$

Here $\ell_{+}^{2}\left(\mathbb{C}^{k}\right)$ denotes the Hilbert space of unilateral square summable sequences of vectors in $\mathbb{C}^{k}$. By $S_{k}$ we denote the unilateral shift on $\ell_{+}^{2}\left(\mathbb{C}^{k}\right)$. For positive integers $k$ and $r$ we write $H_{k \times r}^{\infty}$ for the Banach space of all $k \times r$ matrices with entries from $H^{\infty}$, the algebra of all bounded analytic functions of the open unit disc $\mathbb{D}$. As usual, we identify a $k \times r$ matrix with complex entries with the linear operator from $\mathbb{C}^{r}$ to $\mathbb{C}^{k}$ induced by the action of the matrix on the standard bases. By definition, the infinity norm of $F \in H_{k \times r}^{\infty}$ is given by $\|F\|_{\infty}=\sup _{|z|<1}\|F(z)\|$. A function $F \in H_{k \times r}^{\infty}$ is said to be outer if the Toeplitz operator $T_{F}$ from $\ell_{+}^{2}\left(\mathbb{C}^{r}\right)$ to $\ell_{+}^{2}\left(\mathbb{C}^{k}\right)$ defined by $F$ has a dense range. We call $F \in H_{k \times k}^{\infty}$ invertible outer if $\operatorname{det} F(z) \neq 0$ 
for each $z \in \mathbb{D}$ and $F^{-1}$ belongs to $H_{k \times k}^{\infty}$. Thus $F \in H_{k \times k}^{\infty}$ is invertible outer if and only if $T_{F}$ is invertible, and in that case $T_{F}^{-1}=T_{F^{-1}}$.

\section{The Central COMmutant Lifting SOlution}

In this section we recall the construction of the central solution in the Sz.-NagyFoias commutant lifting theorem, as presented in Chapter IV of [9] with the bound $\gamma$ equal to one. Note that in this setting, by [9. Theorem IV.7.5], the central solution is equal to the maximum entropy solution (see Theorem 2.2 below).

Theorem 2.1 (Commutant lifting). Let $\mathcal{H}^{\prime}$ be an invariant subspace for the backward shift $S_{p}^{*}$ on $\ell_{+}^{2}\left(\mathbb{C}^{p}\right)$ and $T^{\prime}$ the operator on $\mathcal{H}^{\prime}$ obtained by compressing $S_{p}$ to $\mathcal{H}^{\prime}$, that is, $T^{\prime}=P_{\mathcal{H}^{\prime}} S_{p} \mid \mathcal{H}^{\prime}$. Let $\Lambda$ be a contraction mapping $\ell_{+}^{2}\left(\mathbb{C}^{q}\right)$ into $\mathcal{H}^{\prime}$ satisfying $T^{\prime} \Lambda=\Lambda S_{q}$. Then there exists a function $X$ in $H_{p \times q}^{\infty}$ such that

$$
\Lambda=P_{\mathcal{H}^{\prime}} T_{X} \quad \text { and }\|X\|_{\infty} \leq 1 .
$$

Moreover, if $\|\Lambda\|<1$, then a function $X$ in $H_{p \times q}^{\infty}$ satisfying $\Lambda=P_{\mathcal{H}^{\prime}} T_{X}$ and $\|X\|_{\infty} \leq 1$ is given by

$$
\begin{aligned}
& X(z)=U(z) V(z)^{-1}, \\
& U(z)=E_{p}^{*}\left(I-z S_{p}^{*}\right)^{-1} \Lambda\left(I-\Lambda^{*} \Lambda\right)^{-1} E_{q}, \\
& V(z)=E_{q}^{*}\left(I-z S_{q}^{*}\right)^{-1}\left(I-\Lambda^{*} \Lambda\right)^{-1} E_{q} .
\end{aligned}
$$

Moreover, $\operatorname{det} V(z) \neq 0$ for $|z|<1$, the function $V^{-1}$ belongs to $H_{q \times q}^{\infty}$ and is an outer function. In fact, the function $\Theta=V(0)^{1 / 2} V^{-1}$ is the outer spectral factor of the function $I-X^{*} X$, that is

$$
I-X(\zeta)^{*} X(\zeta)=\Theta(\zeta)^{*} \Theta(\zeta), \quad \zeta \in \mathbb{T} \quad \text { a.e.. }
$$

The formulas for $X, U$ and $V$ appearing in (2.2) and the identity (2.3) are obtained from [9, Theorem IV.6.6] using $\gamma=1$ and $A=\Lambda$.

The following theorem (see [9, Theorem IV.7.5]) shows that the function $X$ constructed in the second part of the above theorem is the maximum entropy solution.

Theorem 2.2. The function $X$ in (2.2) is the maximal entropy solution, that is, if $Y$ in $H_{p \times q}^{\infty}$ satisfies $\Lambda=P_{\mathcal{H}^{\prime}} T_{Y}$ and $\|Y\|_{\infty} \leq 1$, then $\mathcal{E}(Y) \leq \mathcal{E}(X)$. Moreover, the maximal entropy solution is unique and

$$
\mathcal{E}(X)=-\ln \operatorname{det}[V(0)]=-\ln \operatorname{det}\left[E_{q}^{*}\left(I-\Lambda^{*} \Lambda\right)^{-1} E_{q}\right] .
$$

In the remaining part of this section we will apply the previous theorems to the special choice of $\Lambda$ associated with our Leech problem. For this special case $\Lambda$ is given in item (iii) of the following lemma for the general case when $G$ and $K$ are matrix-valued $H^{\infty}$ functions and not necessarily rational functions.

Lemma 2.3. Let $G$ and $K$ be matrix-valued $H^{\infty}$ functions of sizes $m \times p$ and $m \times q$, respectively, and assume that $T_{G} T_{G}^{*}-T_{K} T_{K}^{*}$ is strictly positive. Then the following statements hold.

(i) The operator $T_{G} T_{G}^{*}$ is invertible, or equivalently, $T_{G}^{*}$ is one-to-one and has closed range.

(ii) The subspace $\mathcal{H}^{\prime}=\operatorname{Im} T_{G}^{*}$ is invariant for the backward shift $S_{p}^{*}$. 
(iii) The operator $\Lambda=T_{G}^{*}\left(T_{G} T_{G}^{*}\right)^{-1} T_{K}$ viewed as an operator from $\ell_{+}^{2}\left(\mathbb{C}^{q}\right)$ into $\mathcal{H}^{\prime}$ is a strict contraction. Moreover,

$$
T_{G} \Lambda=T_{K} .
$$

(iv) The operator $\Lambda$ intertwines $S_{q}$ with $T^{\prime}$, that is,

$$
T^{\prime} \Lambda=\Lambda S_{q}
$$

where $T^{\prime}$ on $\mathcal{H}^{\prime}$ is the compression of $S_{p}$ to $\mathcal{H}^{\prime}$, that is, $T^{\prime}=P_{\mathcal{H}^{\prime}} S_{p} \mid \mathcal{H}^{\prime}$.

Proof. Because $T_{G} T_{G}^{*}-T_{K} T_{K}^{*}$ is strictly positive, $T_{G} T_{G}^{*}$ is also strictly positive. Hence $T_{G}^{*}$ is one-to-one and has closed range. Thus item (i) holds. From item (i) we conclude that $\mathcal{H}^{\prime}$ is a closed subspace of $\ell_{+}^{2}\left(\mathbb{C}^{p}\right)$. Using $S_{p}^{*} T_{G}^{*}=T_{G}^{*} S_{m}^{*}$, it follows that $\mathcal{H}^{\prime}=\operatorname{Im} T_{G}^{*}$ is an invariant subspace for the backward shift $S_{p}^{*}$. Therefore item (ii) holds.

Using the definition of $\Lambda$ we see that

$$
T_{G} \Lambda u=T_{G} T_{G}^{*}\left(T_{G} T_{G}^{*}\right)^{-1} T_{K} u=T_{K} u, \quad u \in \ell_{+}^{2}\left(\mathbb{C}^{q}\right) .
$$

This proves (2.5). We also have $\Lambda^{*} T_{G}^{*}=T_{K}^{*}$. It follows that the operator

$$
T_{G}\left(I-\Lambda \Lambda^{*}\right) T_{G}^{*}=T_{G} T_{G}^{*}-T_{K} T_{K}^{*}
$$

is strictly positive. Using $\mathcal{H}^{\prime}=\operatorname{Im} T_{G}^{*}$ and the fact that $T_{G}^{*}$ is one-to-one and has closed range, we conclude that $I-\Lambda \Lambda^{*}$ is also strictly positive. In other words, $\Lambda$ is a strict contraction and item (iii) holds.

Recall that $T^{\prime}$ is the compression of $S_{p}$ onto $\mathcal{H}^{\prime}$. Because $\mathcal{H}^{\prime}$ is an invariant subspace for the backward shift $S_{p}^{*}$, we have $P_{\mathcal{H}^{\prime}} S_{p}=T^{\prime} P_{\mathcal{H}^{\prime}}$. (In the language of the commutant lifting theorem, $S_{p}$ is an isometric dilation of $T^{\prime}$.) Notice that $\mathcal{H}^{\prime}=\left(\operatorname{Ker} T_{G}\right)^{\perp}$. Now observe that

$$
T_{G} T^{\prime} \Lambda=T_{G} S_{p} \Lambda=S_{m} T_{G} \Lambda=S_{m} T_{K}=T_{K} S_{q}=T_{G} \Lambda S_{q} .
$$

Hence $T^{\prime} \Lambda=\Lambda S_{q}$. Therefore item (iv) holds.

The above lemma shows that operator $\Lambda=T_{G}^{*}\left(T_{G} T_{G}^{*}\right)^{-1} T_{K}$ mapping $\ell_{+}^{2}\left(\mathbb{C}^{q}\right)$ into $\mathcal{H}^{\prime}$ satisfies the hypothesis of Theorem 2.1. In particular, $X=U V^{-1}$ in (2.2) is the maximal entropy solution; see Theorem 2.2. In Section 5 , we will construct the finite dimensional state space realization for $X$ in Theorem 1.2. According to Theorem 2.1 the operator $\Lambda=P_{\mathcal{H}^{\prime}} T_{X}$ and $\|X\|_{\infty} \leq 1$. Hence $T_{K}=T_{G} \Lambda=T_{G} T_{X}$, or equivalently, $G X=K$. Therefore $X$ is a solution to the Leech problem assocaited to $G$ and $K$. This $X=U V^{-1}$ is also the unique maximal entropy solution over the set of all contractive analytic solutions $Y$ for $G Y=K$. If $Y$ is contractive analytic solution to $G Y=K$, then $T_{G} T_{Y}=T_{K}$ and $\left\|T_{Y}\right\|=\|Y\|_{\infty} \leq 1$. Notice that

$$
T_{G} P_{\mathcal{H}^{\prime}} T_{Y}=T_{G} T_{Y}=T_{K}=T_{G} \Lambda .
$$

Because $\mathcal{H}^{\prime}=\left(\operatorname{Ker} T_{G}\right)^{\perp}$, we have $\Lambda=P_{\mathcal{H}^{\prime}} T_{Y}$. Theorem 2.2 guarantees that $\mathcal{E}(Y) \leq \mathcal{E}(X)$ with equality if and only if $Y=X$. Since $X=U V^{-1}$, formula 1.17) is a direct consequence of Theorem 2.2. 


\section{The infinite Dimensional State SPACE Model}

Throughout this section $G \in H_{m \times p}^{\infty}$ and $K \in H_{m \times q}^{\infty}$, and $T_{G} T_{G}^{*}-T_{K} T_{K}^{*}$ is assumed to be strictly positive. Furthermore, $\Lambda=T_{G}^{*}\left(T_{G} T_{G}^{*}\right)^{-1} T_{K}$ is viewed as an operator from $\ell_{+}^{2}\left(\mathbb{C}^{q}\right)$ into $\mathcal{H}^{\prime}=\operatorname{Im} T_{G}^{*}$, and $X=U V^{-1}$ is the maximal entropy solution in Theorem 2.1 corresponding to this choice of $\Lambda$.

The following proposition provides an infinite dimensional state space realization for $X$ even when $G$ and $K$ are nonrational.

Proposition 3.1. Assume that $T_{G} T_{G}^{*}-T_{K} T_{K}^{*}$ is strictly positive, where $G$ and $K$ are functions in $H_{m \times p}^{\infty}$ and $H_{m \times q}^{\infty}$, respectively. Let $\Lambda$ be the strict contraction defined by $\Lambda=T_{G}^{*}\left(T_{G} T_{G}^{*}\right)^{-1} T_{K}$. Then the function $X=U V^{-1}$ in (2.2), is given by the following infinite dimensional state space realization

$$
X(z)=D_{U} D_{V}^{-1}+z\left(E_{p}^{*} T_{G}^{*}-D_{U} D_{V}^{-1} E_{q}^{*} T_{K}^{*}\right)(I-z F)^{-1} S_{m}^{*} \Xi D_{V}^{-1} \quad(z \in \mathbb{D})
$$

Here $\Xi$ and $F$ are the operators defined by

$$
\begin{aligned}
& \Xi=\left(T_{G} T_{G}^{*}-T_{K} T_{K}^{*}\right)^{-1} T_{K} E_{q}: \mathbb{C}^{q} \rightarrow \ell_{+}^{2}\left(\mathbb{C}^{m}\right), \\
& F=S_{m}^{*}-S_{m}^{*} \Xi D_{V}^{-1} E_{q}^{*} T_{K}^{*} \text { on } \ell_{+}^{2}\left(\mathbb{C}^{m}\right),
\end{aligned}
$$

and $D_{U}$ and $D_{V}$ are given by

$$
D_{U}=E_{p}^{*} T_{G}^{*} \Xi: \mathbb{C}^{q} \rightarrow \mathbb{C}^{p}, \quad D_{V}=I_{q}+E_{q}^{*} T_{K}^{*} \Xi: \mathbb{C}^{q} \rightarrow \mathbb{C}^{q} .
$$

Finally, the spectral radius $r_{\text {spec }}(F) \leq 1$.

The following lemma is used to prove the above result.

Lemma 3.2. Assume that $T_{G} T_{G}^{*}-T_{K} T_{K}^{*}$ is strictly positive, where $G$ and $K$ are functions in $H_{m \times p}^{\infty}$ and $H_{m \times q}^{\infty}$, respectively. Let $\Lambda$ be the strict contraction defined by $\Lambda=T_{G}^{*}\left(T_{G} T_{G}^{*}\right)^{-1} T_{K}$. Then

$$
\begin{aligned}
\left(I-\Lambda^{*} \Lambda\right)^{-1} & =I+T_{K}^{*}\left(T_{G} T_{G}^{*}-T_{K} T_{K}^{*}\right)^{-1} T_{K}, \\
\Lambda\left(I-\Lambda^{*} \Lambda\right)^{-1} & =T_{G}^{*}\left(T_{G} T_{G}^{*}-T_{K} T_{K}^{*}\right)^{-1} T_{K} .
\end{aligned}
$$

Proof. Using $\Lambda=T_{G}^{*}\left(T_{G} T_{G}^{*}\right)^{-1} T_{K}$, we obtain

$$
I-\Lambda^{*} \Lambda=I-T_{K}^{*}\left(T_{G} T_{G}^{*}\right)^{-1} T_{K} .
$$

The operator inversion formula $\left(I-C^{*} A^{-1} C\right)^{-1}=I+C^{*}\left(A-C C^{*}\right)^{-1} C$ yields the formula for $\left(I-\Lambda^{*} \Lambda\right)^{-1}$ in (3.5).

On the other hand,

$$
\begin{aligned}
\Lambda\left(I-\Lambda^{*} \Lambda\right)^{-1} & =T_{G}^{*}\left(T_{G} T_{G}^{*}\right)^{-1} T_{K}\left(I+T_{K}^{*}\left(T_{G} T_{G}^{*}-T_{K} T_{K}^{*}\right)^{-1} T_{K}\right) \\
& =T_{G}^{*}\left(T_{G} T_{G}^{*}\right)^{-1}\left(I+T_{K} T_{K}^{*}\left(T_{G} T_{G}^{*}-T_{K} T_{K}^{*}\right)^{-1}\right) T_{K} \\
& =T_{G}^{*}\left(T_{G} T_{G}^{*}\right)^{-1}\left(\left(T_{G} T_{G}^{*}-T_{K} T_{K}^{*}\right)+T_{K} T_{K}^{*}\right)\left(T_{G} T_{G}^{*}-T_{K} T_{K}^{*}\right)^{-1} T_{K} \\
& =T_{G}^{*}\left(T_{G} T_{G}^{*}-T_{K} T_{K}^{*}\right)^{-1} T_{K} .
\end{aligned}
$$

This yields the formula for $\Lambda\left(I-\Lambda^{*} \Lambda\right)^{-1}$ in (3.6). 
Proof of Proposition 3.1. Recall that $X=U V^{-1}$. We first establish a state space realization for $U$. By employing (3.6), we obtain

$$
\begin{aligned}
U(z) & =E_{p}^{*}\left(I-z S_{p}^{*}\right)^{-1} \Lambda\left(I-\Lambda^{*} \Lambda\right)^{-1} E_{q} \\
& =E_{p}^{*}\left(I-z S_{p}^{*}\right)^{-1} T_{G}^{*}\left(T_{G} T_{G}^{*}-T_{K} T_{K}^{*}\right)^{-1} T_{K} E_{q} \\
& =E_{p}^{*} T_{G}^{*}\left(I-z S_{m}^{*}\right)^{-1}\left(T_{G} T_{G}^{*}-T_{K} T_{K}^{*}\right)^{-1} T_{K} E_{q} \\
& =E_{p}^{*} T_{G}^{*}\left(I-z S_{m}^{*}\right)^{-1} \Xi, \quad|z|<1 .
\end{aligned}
$$

See (3.2) for the definition of $\Xi$. Using $D_{U}=E_{p}^{*} T_{G}^{*} \Xi$, we see that a state space realization for $U$ is given by

$$
U(z)=D_{U}+z E_{p}^{*} T_{G}^{*}\left(I-z S_{m}^{*}\right)^{-1} S_{m}^{*} \Xi, \quad|z|<1 .
$$

To compute a state space realization for $V$, we use (3.5) in the following calculation:

$$
\begin{aligned}
V(z) & =E_{q}^{*}\left(I-z S_{q}^{*}\right)^{-1}\left(I-\Lambda^{*} \Lambda\right)^{-1} E_{q} \\
& =E_{q}^{*} E_{q}+E_{q}^{*}\left(I-z S_{q}^{*}\right)^{-1} T_{K}^{*}\left(T_{G} T_{G}^{*}-T_{K} T_{K}^{*}\right)^{-1} T_{K} E_{q} \\
& =I_{q}+E_{q}^{*} T_{K}^{*}\left(I-z S_{m}^{*}\right)^{-1} \Xi \\
& =I_{q}+E_{q}^{*} T_{K}^{*} \Xi+z E_{q} T_{K}^{*}\left(I-z S_{m}^{*}\right)^{-1} S_{m}^{*} \Xi, \quad|z|<1 .
\end{aligned}
$$

By consulting (3.4), we see that a state space realization for $V$ is given by

$$
V(z)=D_{V}+z E_{q}^{*} T_{K}^{*}\left(I-z S_{m}^{*}\right)^{-1} S_{m}^{*} \Xi, \quad|z|<1 .
$$

Using a classical state space inversion formula, the inverse of $V(z)^{-1}$ in a neighborhood of zero is given by

$$
\begin{gathered}
V(z)^{-1}=D_{V}^{-1}-z D_{V}^{-1} E_{q}^{*} T_{K}^{*}(I-z F)^{-1} S_{m}^{*} \Xi D_{V}^{-1}, \text { where } \\
F=S_{m}^{*}-S_{m}^{*} \Xi D_{V}^{-1} E_{q}^{*} T_{K}^{*}, \text { as in (3.3). }
\end{gathered}
$$

On the other hand, by the final part of Theorem 2.1. we know that $V(z)$ is invertible for each $z$ in $\mathbb{D}$. Since $r_{\text {spec }}\left(S_{m}^{*}\right) \leq 1$, we can then apply Theorem 2.1 in [4] (with $\lambda=z^{-1}$ ) to show that $r_{\text {spec }}(F) \leq 1$. Thus

$$
V(z)^{-1}=D_{V}^{-1}-z D_{V}^{-1} E_{q}^{*} T_{K}^{*}(I-z F)^{-1} S_{m}^{*} \Xi D_{V}^{-1}, \quad|z|<1 .
$$

To compute a state space realization for the maximum entropy solution $X$, we first observe (using the identity (3.3)) that

$$
\begin{aligned}
- & z E_{p}^{*} T_{G}^{*}\left(I-z S_{m}^{*}\right)^{-1} S_{m}^{*} \Xi D_{V}^{-1} E_{q}^{*} T_{K}^{*}(I-z F)^{-1} S_{m}^{*} \Xi D_{V}^{-1} \\
& =z E_{p}^{*} T_{G}^{*}\left(I-z S_{m}^{*}\right)^{-1}\left(F-S_{m}^{*}\right)(I-z F)^{-1} S_{m}^{*} \Xi D_{V}^{-1} \\
& =E_{p}^{*} T_{G}^{*}\left(I-z S_{m}^{*}\right)^{-1}\left(\left(I-z S_{m}^{*}\right)-(I-z F)\right)(I-z F)^{-1} S_{m}^{*} \Xi D_{V}^{-1} \\
& =E_{p}^{*} T_{G}^{*}(I-z F)^{-1} S_{m}^{*} \Xi D_{V}^{-1}-E_{p}^{*} T_{G}^{*}\left(I-z S_{m}^{*}\right)^{-1} S_{m}^{*} \Xi D_{V}^{-1} .
\end{aligned}
$$

This readily implies that

$$
\begin{aligned}
& -z^{2} E_{p}^{*} T_{G}^{*}\left(I-z S_{m}^{*}\right)^{-1} S_{m}^{*} \Xi D_{V}^{-1} E_{q}^{*} T_{K}^{*}(I-z F)^{-1} S_{m}^{*} \Xi D_{V}^{-1}= \\
& \quad=z E_{p}^{*} T_{G}^{*}(I-z F)^{-1} S_{m}^{*} \Xi D_{V}^{-1}-z E_{p}^{*} T_{G}^{*}\left(I-z S_{m}^{*}\right)^{-1} S_{m}^{*} \Xi D_{V}^{-1} .
\end{aligned}
$$


The state space realizations for $U$ in (3.7) and $V^{-1}$ in (3.9) then yield:

$$
\begin{aligned}
U(z) V(z)^{-1}= & D_{U} D_{V}^{-1}-z D_{U} D_{V}^{-1} E_{q}^{*} T_{K}^{*}(I-z F)^{-1} S_{m}^{*} \Xi D_{V}^{-1} \\
& +z E_{p}^{*} T_{G}^{*}\left(I-z S_{m}^{*}\right)^{-1} S_{m}^{*} \Xi D_{V}^{-1} \\
& -z^{2} E_{p}^{*} T_{G}^{*}\left(I-z S_{m}^{*}\right)^{-1} S_{m}^{*} \Xi D_{V}^{-1} E_{q}^{*} T_{K}^{*}(I-z F)^{-1} S_{m}^{*} \Xi D_{V}^{-1} \\
= & D_{U} D_{V}^{-1}+z\left(E_{p}^{*} T_{G}^{*}-D_{U} D_{V}^{-1} E_{q} T_{K}^{*}\right)(I-z F)^{-1} S_{m}^{*} \Xi D_{V}^{-1}
\end{aligned}
$$

when $z \in \mathbb{D}$. This proves the state space formula for $X$ in (3.1).

\section{Proof of Theorem 1.1}

Throughout the section $G$ and $K$ are stable rational matrix functions of sizes $m \times p$ and $m \times q$, respectively, and we assume that $\left[\begin{array}{ll}G & K\end{array}\right]$ is given by the observable stable realization (1.3). We first prove two lemmas. The first deals with the $m \times m$ rational matrix function $R$ defined by (1.5).

Lemma 4.1. Let $R$ be the $m \times m$ rational matrix function defined by (1.5). Then $T_{R}$ is strictly positive whenever $T_{G} T_{G}^{*}-T_{K} T_{K}^{*}$ is strictly positive.

Proof. Assume that $T_{G} T_{G}^{*}-T_{K} T_{K}^{*}$ is strictly positive. For each $z \in \mathbb{D}$ put $\varphi_{z, m}=$ $\left[\begin{array}{llll}I_{m} & z I_{m} & z^{2} I_{m} & \cdots\end{array}\right]^{*}$. Note that

$$
T_{G}^{*} \varphi_{z, m}=\varphi_{z, p} G(z)^{*}, \quad T_{K}^{*} \varphi_{z, m}=\varphi_{z, q} K(z)^{*}, \quad \varphi_{z, m}^{*} \varphi_{z, m}=\frac{1}{1-|z|^{2}} I_{m} .
$$

Since $T_{G} T_{G}^{*}-T_{K} T_{K}^{*}$ is assumed to be strictly positive, there exists an $\eta>0$ such that $T_{G} T_{G}^{*}-T_{K} T_{K}^{*} \geq \eta I$. Multiplying this inequality by $\varphi_{z, m}$ on the right and by $\varphi_{z, m}^{*}$ on the left gives

$$
\frac{G(z) G(z)^{*}-K(z) K(z)^{*}}{1-|z|^{2}} \geq \frac{\eta}{1-|z|^{2}} I_{m} \quad(z \in \mathbb{D}) .
$$

Multiplying with $1-|z|^{2}$ and taking limits $z \rightarrow e^{i \omega}$ on the unit circle, shows

$$
R\left(e^{i \omega}\right)=G\left(e^{i \omega}\right) G\left(e^{i \omega}\right)^{*}-K\left(e^{i \omega}\right) K\left(e^{i \omega}\right)^{*} \geq \eta I_{m}, \quad 0 \leq \omega \leq 2 \pi .
$$

This implies $T_{R} \geq \eta I_{m}$.

Lemma 4.2. Let $W_{\text {obs }}$ be defined by (1.4), and let $P_{1}$ and $P_{2}$ be the unique $n \times n$ matrix solutions of the Stein equations (1.9). Then

$$
T_{G} T_{G}^{*}-T_{K} T_{K}^{*}=T_{R}+W_{o b s}\left(P_{2}-P_{1}\right) W_{o b s}^{*} .
$$

In particular, the operator $T_{G} T_{G}^{*}-T_{K} T_{K}^{*}$ is strictly positive if and only if the operator $T_{R}+W_{o b s}\left(P_{2}-P_{1}\right) W_{o b s}^{*}$ is strictly positive.

Proof. We first recall some elementary facts concerning Hankel operators. To this end, let

$$
H_{G}=\left[\begin{array}{cccc}
G_{1} & G_{2} & G_{3} & \cdots \\
G_{2} & G_{3} & G_{4} & \cdots \\
G_{4} & G_{5} & G_{6} & \cdots \\
\vdots & \vdots & \vdots & \vdots
\end{array}\right]: \ell_{+}^{2}\left(\mathbb{C}^{p}\right) \rightarrow \ell_{+}^{2}\left(\mathbb{C}^{m}\right)
$$

be the Hankel operator determined by the Taylor series $G(z)=\sum_{\nu=0}^{\infty} z^{\nu} G_{\nu}$. In a similar way, let $H_{K}$ be the corresponding Hankel operator mapping $\ell_{+}^{2}\left(\mathbb{C}^{q}\right)$ into 
$\ell_{+}^{2}\left(\mathbb{C}^{m}\right)$ determined by $K$. Let $W_{\text {con }, 1}$ mapping $\ell_{+}^{2}\left(\mathbb{C}^{p}\right)$ into $\mathbb{C}^{n}$ and $W_{\text {con }, 2}$ mapping $\ell_{+}^{2}\left(\mathbb{C}^{q}\right)$ into $\mathbb{C}^{n}$ be the controllability operators defined by

$$
W_{c o n, j}=\left[\begin{array}{lllll}
B_{j} & A B_{j} & A^{2} B_{j} & A^{3} B_{j} & \cdots
\end{array}\right], \quad j=1,2 .
$$

From (1.9) we see that $P_{j}=W_{c o n, j} W_{c o n, j}^{*}$ for $j=1,2$. Using $G_{\nu}=C A^{\nu-1} B_{1}$ for all integers $\nu \geq 1$ and the corresponding result for $K$, we see that $H_{G}=W_{o b s} W_{c o n, 1}$ and $H_{K}=W_{o b s} W_{c o n, 1}$. Finally,

$$
H_{G} H_{G}^{*}=W_{o b s} P_{1} W_{o b s}^{*} \quad \text { and } \quad H_{K} H_{K}^{*}=W_{o b s} P_{2} W_{o b s}^{*} .
$$

Next, notice the Toeplitz operators $T_{G G^{*}}$ and $T_{K K^{*}}$ are given by the following identities:

$$
T_{G G^{*}}=T_{G} T_{G}^{*}+H_{G} H_{G}^{*} \quad \text { and } \quad T_{K K^{*}}=T_{K} T_{K}^{*}+H_{K} H_{K}^{*} .
$$

Using $R=G G^{*}-K K^{*}$, we have $T_{R}=T_{G} T_{G}^{*}-T_{K} T_{K}^{*}+H_{G} H_{G}^{*}-H_{K} H_{K}^{*}$. But then (4.2) yields (4.1).

Proof of Theorem 1.1. Assume the operator $T_{G} T_{G}^{*}-T_{K} T_{K}^{*}$ is strictly positive. Then Lemma 4.1 tells us $T_{R}$ is strictly positive, and hence, see Remark 1.3. item (i) in Theorem 1.1 is fulfilled. Furthermore, applying Lemma 4.3 below with

$$
M=T_{G} T_{G}^{*}-T_{K} T_{K}^{*}, \quad T=T_{R}, \quad W=W_{o b s} \quad \text { and } \quad N=P_{2}-P_{1},
$$

noting that $M=T+W N W^{*}$ is strictly positive, by the identity (4.1), we see that the matrix $Q^{-1}+P_{2}-P_{1}$ is strictly positive, and hence item (ii) in Theorem 1.1 is fulfilled. Furthermore, again in view of (4.1), in this case the inversion formula (4.5) yields the formula to compute the inverse of $T_{G} T_{G}^{*}-T_{K} T_{K}^{*}$ in (1.11).

Conversely, assume items (i) and (ii) in Theorem 1.1 are satisfied. Then item (i) implies that $T_{R}$ is strictly positive, as explained in Remark 1.3 and $Q=$ $W_{o b s}^{*} T_{R}^{-1} W_{o b s}=W^{*} T^{-1} W$, using the notation of (4.3) in the last identity. Note that item (ii) states that $Q^{-1}-N=Q^{-1}-P_{1}+P_{2}$ is strictly positive. Hence again using Lemma 4.3 below and the identity (4.1), we see that item (ii) implies that $T_{G} T_{G}^{*}-T_{K} T_{K}^{*}$ is strictly positive.

Lemma 4.3. Let $M$ be an operator acting on a Hilbert space $\mathcal{H}$ such that

$$
M=T+W N W^{*},
$$

where $T$ on $\mathcal{H}$ is a strictly positive operator, $N$ is a self adjoint operator on a Hilbert space $\mathcal{X}$, and $W$ is an operator mapping $\mathcal{X}$ into $\mathcal{H}$ which is one-to-one and has closed range. Set $Q=W^{*} T^{-1} W$. Then $Q$ is invertible. Furthermore, $M$ is strictly positive if and only if $Q^{-1}+N$ is strictly positive. Moreover, in that case,

$$
M^{-1}=T^{-1}-T^{-1} W N(I+Q N)^{-1} W^{*} T^{-1} .
$$

Proof. Replacing $M$ by $T^{-1 / 2} M T^{-1 / 2}$ and $W$ by $T^{-1 / 2} W$, we see that without loss of generality we may assume that $T$ is the identity operator on $\mathcal{H}$. Therefore, in what follows $M=I+W N W^{*}$. Note that in this case $Q=W^{*} W$.

The fact that $W$ is one-to-one and has closed range, implies that $Q=W^{*} W$ is invertible. It follows that the Moore-Penrose left inverse $W^{+}$of $W$ is well-defined and is given by $W^{+}=\left(W^{*} W\right)^{-1} W^{*}=Q^{-1} W^{*}$. Furthermore, the orthogonal project $\mathbb{P}$ on $\mathcal{H}$ mapping $\mathcal{H}$ onto the range of $W$ is given $\mathbb{P}=W Q^{-1} W^{*}$. Now note that

$$
M=I+W N W^{*}=I-\mathbb{P}+\mathbb{P}+W N W^{*}=I-\mathbb{P}+W\left(Q^{-1}+N\right) W^{*} .
$$


Put $\mathcal{H}_{1}=\operatorname{Im} W$ and $\mathcal{H}_{0}=\operatorname{Ker} W^{*}$, and consider the operators

$$
\begin{aligned}
& \tau_{1}: \mathcal{H}_{1} \rightarrow \mathcal{H}, \quad \tau_{1} u=u \quad\left(u \in \mathcal{H}_{1}\right), \\
& W_{1}: \mathcal{X} \rightarrow \mathcal{H}_{1}, \quad W_{1} x=W x \quad(x \in \mathcal{X}) .
\end{aligned}
$$

Note that $W=\tau_{1} W_{1}$ and $W^{*}=W_{1}^{*} \tau_{1}^{*}$. Furthermore, $W_{1}$ is invertible, and $W_{1}^{-1}=$ $Q^{-1} W^{*} \tau_{1}$. Using (4.6) we see that relative to the orthogonal decomposition $\mathcal{H}=$ $\mathcal{H}_{0} \oplus \mathcal{H}_{1}$ the operator $M$ admits the following block operator matrix representation:

$$
M=\left[\begin{array}{cc}
I_{\mathcal{H}} & 0 \\
0 & W_{1}\left(Q^{-1}+N\right) W_{1}^{*}
\end{array}\right]
$$

Since $W_{1}$ is invertible, it follows that $M$ is strictly positive if and only $Q^{-1}+N$ is strictly positive. Moreover, in that case

$$
\begin{aligned}
M^{-1} & =\left[\begin{array}{cc}
I_{\mathcal{H}_{0}} & 0 \\
0 & W_{1}^{-*}\left(Q^{-1}+N\right)^{-1} W_{1}^{-1}
\end{array}\right] \\
& =\left[\begin{array}{cc}
I_{\mathcal{H}_{0}} & 0 \\
0 & \tau_{1}^{*} W Q^{-1}\left(Q^{-1}+N\right)^{-1} Q^{-1} W^{*} \tau_{1}
\end{array}\right]
\end{aligned}
$$

It follows that

$$
\begin{aligned}
M^{-1} & =I-\mathbb{P}+W Q^{-1}\left(Q^{-1}+N\right)^{-1} Q^{-1} W^{*} \\
& =I-W Q^{-1} W^{*}+W Q^{-1}\left(Q^{-1}+N\right)^{-1} Q^{-1} W^{*} \\
& =I-W\left(Q^{-1}-Q^{-1}\left(Q^{-1}+N\right)^{-1} Q^{-1}\right) W^{*}
\end{aligned}
$$

Finally, note that

$$
\begin{aligned}
& Q^{-1}-Q^{-1}\left(Q^{-1}+N\right)^{-1} Q^{-1}= \\
& \quad=Q^{-1}-\left(Q^{-1}+N-N\right)\left(Q^{-1}+N\right)^{-1} Q^{-1} \\
& \quad=N\left(Q^{-1}+N\right)^{-1} Q^{-1}=N(I+Q N)^{-1} .
\end{aligned}
$$

This proves (4.5).

For a version of Lemma 4.3 with $T$ just nonnegative, not necessarily strictly positive, see Lemma 2.10 in [11.

\section{Proof of Theorem 1.2}

In this section, we will convert the infinite dimensional state space realization for the central solution $X$ in (3.1) to the finite dimensional realization for $X$ in (1.12), and in the mean time prove Theorem 1.2. Throughout $G$ and $K$ are the rational matrix functions described by the observable stable realization (1.3), and we assume that $T_{G} T_{G}^{*}-T_{K} T_{K}^{*}$ is strictly positive. Thus items (i) and (ii) in Theorem 1.1 are satisfied. In what follows we shall freely use the notations introduced in these two items. In particular, the operator $T_{R}$ is strictly positive, $Q$ is the stabilizing solution of the algebraic Riccati equation (1.10), and the matrix $\Delta=R_{0}-\Gamma^{*} Q \Gamma$ is strictly positive. We set

$$
C_{0}=\Delta^{-1}\left(C-\Gamma^{*} Q A\right) \text { and } A_{0}=A-\Gamma C_{0} .
$$

By item (c) in Theorem 1.1 the matrix $A_{0}$ is stable. Using $C_{0}$ and $A_{0}$ in (5.1), the Riccati equation (1.10) can be rewritten as a Stein equation:

$$
Q-A^{*} Q A_{0}=C^{*} C_{0} .
$$


The observability operator for the pair $\left\{C_{0}, A_{0}\right\}$ is the operator $W_{0}$ defined by

$$
W_{0}=\left[\begin{array}{c}
C_{0} \\
C_{0} A_{0} \\
C_{0} A_{0}^{2} \\
\vdots
\end{array}\right]: \mathbb{C}^{n} \rightarrow \ell_{+}^{2}\left(\mathbb{C}^{m}\right) .
$$

We need the following lemma (cf., identity (3.19) in [13]):

Lemma 5.1. The operator $W_{0}$ is one-to-one, $T_{R}^{-1} W_{o b s}=W_{0}$, and

$$
R(z) C_{0}\left(I_{n}-z A_{0}\right)^{-1}=C\left(I_{n}-z A\right)^{-1}+\Gamma^{*}\left(z I_{n}-A^{*}\right)^{-1} Q .
$$

Proof. Let us assume that (5.4) has been proved. Note that the matrix function $C\left(I_{n}-z A\right)^{-1}$ is a stable rational function, while $\Gamma^{*}\left(z I_{n}-A^{*}\right)^{-1} Q$ is a rational function which is analytic on the exterior of the open unit disc and has the value zero at infinity. But then (5.4) implies that $T_{R} W_{0} x$ is equal to $W_{\text {obs }} x$ for each $x \in \mathbb{C}^{n}$. Since $T_{R}$ is invertible, we get $W_{0}=T_{R}^{-1} W_{\text {obs }}$. Recall that $W_{\text {obs }}$ is one to one. Therefore $W_{0}=T_{R}^{-1} W_{o b s}$ is also one to one.

It remains to prove (5.4). To do this we use the realization (1.6). Using $\Gamma C_{0}=$ $A-A_{0}$, a standard calculation shows that

$$
z C\left(I_{n}-z A\right)^{-1} \Gamma C_{0}\left(I_{n}-z A_{0}\right)^{-1}=C\left(I_{n}-z A\right)^{-1}-C\left(I_{n}-z A_{0}\right)^{-1} .
$$

Analogously, using the Stein equation (5.2), one computes that

$$
\begin{aligned}
\Gamma^{*}\left(z I_{n}-A^{*}\right)^{-1} C^{*} C_{0}\left(I_{n}-z A_{0}\right)^{-1}= & \Gamma^{*}\left(z I_{n}-A^{*}\right)^{-1} Q+ \\
& +\Gamma^{*} Q A_{0}\left(I_{n}-z A_{0}\right)^{-1} .
\end{aligned}
$$

Using the realization (1.6) the two preceding identities yield

$$
\begin{aligned}
R(z) C_{0}\left(I_{n}-z A_{0}\right)^{-1}=C\left(I_{n}-z A\right)^{-1} & +\Gamma^{*}\left(z I_{n}-A^{*}\right)^{-1} Q+ \\
& +\left(-C+R_{0} C_{0}+\Gamma^{*} Q A_{0}\right)\left(I_{n}-z A_{0}\right)^{-1} .
\end{aligned}
$$

Next using the two identities in (5.1) we see that

$$
\begin{aligned}
-C+R_{0} C_{0}+\Gamma^{*} Q A_{0} & =-C+R_{0} C_{0}+\Gamma^{*} Q A-\Gamma^{*} Q \Gamma C_{0} \\
& =-\left(C-\Gamma^{*} Q A\right)+\left(R_{0}-\Gamma^{*} Q \Gamma\right) C_{0} \\
& =-\left(C-\Gamma^{*} Q A\right)+\Delta C_{0}=0 .
\end{aligned}
$$

This proves (5.4).

Proof of Theorem 1.2. In the course of this proof we shall often use the following identity (which follows from (1.18) and Lemma 5.1):

$$
W_{o b s}^{*} W_{0}=Q .
$$

The Schur complement for $T_{R}$. From the realization (1.6) it follows that $T_{R}$ admits a block $2 \times 2$ matrix representation

$$
T_{R}=\left[\begin{array}{cc}
R_{0} & \Gamma^{*} W_{o b s}^{*} \\
W_{\text {obs }} \Gamma & T_{R}
\end{array}\right] \text { on }\left[\begin{array}{c}
\mathbb{C}^{m} \\
\ell_{+}^{2}\left(\mathbb{C}^{m}\right)
\end{array}\right] .
$$

Since $T_{R}$ is invertible, the Schur complement with respect to the $(2,2)$ entry is given by

$$
R_{0}-\Gamma^{*} W_{o b s}^{*} T_{R}^{-1} W_{o b s} \Gamma=R_{0}-\Gamma^{*} Q \Gamma=\Delta .
$$


It follows (see. e.g., [4, page 29]) that the inverse of $T_{R}$ is given by

$$
T_{R}^{-1}=\left[\begin{array}{cc}
\Delta^{-1} & -\Delta^{-1} \Gamma^{*} W_{o b s}^{*} T_{R}^{-1} \\
-T_{R}^{-1} W_{o b s} \Gamma \Delta^{-1} & T_{R}^{-1}+T_{R}^{-1} W_{o b s} \Gamma \Delta^{-1} \Gamma^{*} W_{o b s}^{*} T_{R}^{-1}
\end{array}\right] \text { on }\left[\begin{array}{c}
\mathbb{C}^{m} \\
\ell_{+}^{2}\left(\mathbb{C}^{m}\right)
\end{array}\right]
$$

A matrix representation for $\Xi$. To compute a finite dimensional realization for our central (maximum entropy) solution $X$ in (3.1), we need a formula for $\Xi$ involving the state space data. Note that (1.3) is equivalent to the following two realizations:

$$
G(z)=D_{1}+z C\left(I_{n}-z A\right)^{-1} B_{1}, \quad K(z)=D_{2}+z C\left(I_{n}-z A\right)^{-1} B_{2} .
$$

Using the realization for $K$ in (5.6) and (1.11), we obtain

$$
\begin{aligned}
\Xi & =\left(T_{G} T_{G}^{*}-T_{K} T_{K}^{*}\right)^{-1} T_{K} E_{q} \\
& =T_{R}^{-1} T_{K} E_{q}+T_{R}^{-1} W_{o b s} \Omega W_{o b s}^{*} T_{R}^{-1} T_{K} E_{q} \quad \text { [by (1.11)] } \\
& =T_{R}^{-1} T_{K} E_{q}+W_{0} \Omega W_{0}^{*} T_{K} E_{q} \quad[\text { by Lemma [5.1] } \\
& =T_{R}^{-1} T_{K} E_{q}+W_{0} \Omega\left[\begin{array}{ll}
C_{0}^{*} & A_{0}^{*} W_{0}^{*}
\end{array}\right]\left[\begin{array}{c}
D_{2} \\
W_{o b s} B_{2}
\end{array}\right] \\
& =T_{R}^{-1} T_{K} E_{q}+W_{0} \Omega\left(C_{0}^{*} D_{2}+A_{0}^{*} Q B_{2}\right) \quad[\text { by (5.5) }] \\
& =T_{R}^{-1} T_{K} E_{q}+W_{0} \Omega C_{2}^{*} \quad\left[\text { with } C_{2} \text { as in [1.13) }\right] .
\end{aligned}
$$

To compute $T_{R}^{-1} T_{K} E_{q}$, we use the $2 \times 2$ operator matrix representation of $T_{R}^{-1}$ given above. This yields

$$
\begin{aligned}
T_{R}^{-1} T_{K} E_{q} & =\left[\begin{array}{cc}
\Delta^{-1} & -\Delta^{-1} \Gamma^{*} W_{o b s}^{*} T_{R}^{-1} \\
-T_{R}^{-1} W_{o b s} \Gamma \Delta^{-1} & T_{R}^{-1}+T_{R}^{-1} W_{o b s} \Gamma \Delta^{-1} \Gamma^{*} W_{o b s}^{*} T_{R}^{-1}
\end{array}\right]\left[\begin{array}{c}
D_{2} \\
W_{o b s} B_{2}
\end{array}\right] \\
& =\left[\begin{array}{c}
\Delta^{-1} D_{2}-\Delta^{-1} \Gamma^{*} Q B_{2} \\
W_{0}\left(B_{2}+\Gamma \Delta^{-1} \Gamma^{*} Q B_{2}-\Gamma \Delta^{-1} D_{2}\right)
\end{array}\right] .
\end{aligned}
$$

Substituting this into our previous formula for $\Xi$ we arrive at

$$
\Xi=\left[\begin{array}{c}
\Delta^{-1} D_{2}-\Delta^{-1} \Gamma^{*} Q B_{2}+C_{0} \Omega C_{2}^{*} \\
W_{0}\left(B_{2}+\Gamma \Delta^{-1} \Gamma^{*} Q B_{2}-\Gamma \Delta^{-1} D_{2}+A_{0} \Omega C_{2}^{*}\right)
\end{array}\right]=\left[\begin{array}{c}
D_{0} \\
W_{0} B_{0}
\end{array}\right] .
$$

See Theorem 1.2 for the definitions of $D_{0}$ and $B_{0}$. Finally, note that the identity (5.7) also shows that $S_{m}^{*} \Xi=W_{0} B_{0}$.

The state space operator $F$. The state space operator

$$
F=S_{m}^{*}-S_{m}^{*} \Xi D_{V}^{-1} E_{q}^{*} T_{K}^{*}=S_{m}^{*}-W_{0} B_{0} D_{V}^{-1} E_{q}^{*} T_{K}^{*} .
$$

The state space realizations for $G$ and $K$ in (5.6) yield

$$
\begin{aligned}
& E_{p}^{*} T_{G}^{*} W_{0}=\left[\begin{array}{ll}
D_{1}^{*} & B_{1}^{*} W_{o b s}^{*}
\end{array}\right]\left[\begin{array}{c}
C_{0} \\
W_{0} A_{0}
\end{array}\right]=D_{1}^{*} C_{0}+B_{1}^{*} Q A_{0}=C_{1}, \\
& E_{p}^{*} T_{K}^{*} W_{0}=\left[\begin{array}{ll}
D_{2}^{*} & B_{2}^{*} W_{o b s}^{*}
\end{array}\right]\left[\begin{array}{c}
C_{0} \\
W_{0} A_{0}
\end{array}\right]=D_{2}^{*} C_{0}+B_{2}^{*} Q A_{0}=C_{2} .
\end{aligned}
$$

Using the definition of $C_{2}$ with $S_{m}^{*} W_{0}=W_{0} A_{0}$, we obtain

$$
F W_{0}=S_{m}^{*} W_{0}-W_{0} B_{0} D_{V}^{-1} E_{q} T_{K}^{*} W_{0}=W_{0} A_{0}-W_{0} B_{0} D_{V}^{-1} C_{2} .
$$

This leads to the following intertwining relation:

$$
F W_{0}=W_{0} A^{\times} \quad \text { where } \quad A^{\times}=A_{0}-B_{0} D_{V}^{-1} C_{2} .
$$

This readily implies that

$$
(I-z F)^{-1} S_{m}^{*} \Xi=(I-z F)^{-1} W_{0} B_{0}=W_{0}\left(I_{n}-z A^{\times}\right)^{-1} B_{0} .
$$


Substituting the previous formulas into our state space formula for $X$ in (3.1), we obtain

$$
\begin{aligned}
X(z) & =D_{U} D_{V}^{-1}+z\left(E_{p}^{*} T_{G}^{*}-D_{U} D_{V}^{-1} E_{q}^{*} T_{K}^{*}\right)(I-z F)^{-1} S_{m}^{*} \Xi D_{V}^{-1} \\
& =D_{U} D_{V}^{-1}+z\left(E_{p}^{*} T_{G}^{*}-D_{U} D_{V}^{-1} E_{q}^{*} T_{K}^{*}\right) W_{0}\left(I_{n}-z A^{\times}\right)^{-1} B_{0} D_{V}^{-1} \\
& =D_{U} D_{V}^{-1}+z\left(C_{1}-D_{U} D_{V}^{-1} C_{2}\right)\left(I_{n}-z A^{\times}\right)^{-1} B_{0} D_{V}^{-1} .
\end{aligned}
$$

Computing $D_{U}$ and $D_{V}$. To complete our finite dimensional state space realization formula for $X$ in (1.12), we need an expression for $D_{U}$ and $D_{V}$, that is,

$$
\begin{aligned}
& D_{U}=E_{p}^{*} T_{G}^{*} \Xi=\left[\begin{array}{ll}
D_{1}^{*} & B_{1}^{*} W_{o b s}^{*}
\end{array}\right]\left[\begin{array}{c}
D_{0} \\
W_{0} B_{0}
\end{array}\right]=D_{1}^{*} D_{0}+B_{1}^{*} Q B_{0} \\
& D_{V}=I_{q}+E_{q}^{*} T_{K}^{*} \Xi=I_{q}+\left[\begin{array}{ll}
D_{2}^{*} & B_{2}^{*} W_{o b s}^{*}
\end{array}\right]\left[\begin{array}{c}
D_{0} \\
W_{0} B_{0}
\end{array}\right]=I+D_{2}^{*} D_{0}+B_{2}^{*} Q B_{0} .
\end{aligned}
$$

This together with (5.12) yields the finite dimensional state space formula for the maximum entropy solution $X$ in (1.12).

It is noted that $V(0)=D_{V}$. So the entropy $\mathcal{E}(X)=-\ln \operatorname{det}\left[D_{V}\right]$; see (2.4).

The function $V$ is invertible outer and $A^{\times}$is stable. Recall that $S_{m}^{*} \Xi=W_{0} B_{0}$. Using this with $S_{m}^{*} W_{0}=W_{0} A_{0}$ and the state space realization for $V$ in (3.8), we obtain

$$
\begin{aligned}
V(z) & =D_{V}+z E_{q}^{*} T_{K}^{*}\left(I-z S_{m}^{*}\right)^{-1} S_{m}^{*} \Xi \\
& =D_{V}+z E_{q}^{*} T_{K}^{*}\left(I-z S_{m}^{*}\right)^{-1} W_{0} B_{0} \\
& =D_{V}+z E_{q}^{*} T_{K}^{*} W_{0}\left(I_{n}-z A_{0}\right)^{-1} B_{0} .
\end{aligned}
$$

Since $C_{2}=E_{q}^{*} T_{K}^{*} W_{0}$, a finite dimensional realization for $V$ is given by

$$
V(z)=D_{V}+z C_{2}\left(I_{n}-z A_{0}\right)^{-1} B_{0} .
$$

Since $A_{0}$ is stable, the function $V$ is a stable rational matrix function. In particular, $V$ belongs to $H_{q \times q}^{\infty}$. From Theorem 2.1 we know that $V^{-1}$ belongs to $H_{q \times q}^{\infty}$. Thus both $V$ and $V^{-1}$ are in $H_{q \times q}^{\infty}$, and so $V$ is invertible outer.

Next we prove that $A^{\times}$is stable. By employing a standard state space inversion formula, the inverse for $V$ given by

$$
V(z)^{-1}=D_{V}^{-1}-z D_{V}^{-1} C_{2}\left(I_{n}-z A^{\times}\right)^{-1} B_{0} D_{V}^{-1}
$$

where $A^{\times}=A_{0}-B_{0} D_{V}^{-1} C_{2}$. We already know that $V^{-1}$ belongs to $H_{q \times q}^{\infty}$. Hence $V^{-1}$ is also a stable rational matrix function. Because $A_{0}$ is stable and $V(z)^{-1}$ is analytic in the closed unit disc, Theorem 2.1 in [4] tells us that $A^{\times}$is stable.

The solution $X$ is strictly contractive. It remains to show that $\|X\|_{\infty}<1$. From Theorem 2.1 we know that $\Theta=V(0) V^{-1}$ is the outer spectral factor of $I-X^{*} X$. However, as proved in the preceding paragraph, the function $V^{-1}$ is invertible outer. Hence $\Theta$ is invertible outer. The latter implies that $I-X^{*}(\zeta) X(\zeta)$ is strictly positive for each $\zeta \in \mathbb{T}$. Therefore $\|X(\zeta)\|<1$ for $\zeta \in \mathbb{T}$. Thus $X$ is a strictly contractive solution to our Leech problem.

Remark 5.2. For later purposes (see the next remark) we mention that $U$ is given by the following finite dimensional realization:

$$
U(z)=D_{U}+z C_{1}\left(I_{n}-z A_{0}\right)^{-1} B_{0} .
$$


The proof is similar to the proof of the realization of $V$ in (5.13). Indeed, using $S_{m}^{*} W_{0}=W_{0} A_{0}$ with the state space realization for $U$ in (3.7), we obtain

$$
\begin{aligned}
U(z) & =D_{U}+z E_{p}^{*} T_{G}^{*}\left(I-z S_{m}^{*}\right)^{-1} S_{m}^{*} \Xi \\
& =D_{U}+z E_{p}^{*} T_{G}^{*}\left(I-z S_{m}^{*}\right)^{-1} W_{0} B_{0} \\
& =D_{U}+z E_{p}^{*} T_{G}^{*} W_{0}\left(I_{n}-z A_{0}\right)^{-1} B_{0} .
\end{aligned}
$$

Remark 5.3. Given the various matrices appearing in Theorems 1.1 and 1.2 one can now also prove directly that the function $X=U V^{-1}$ given by (1.12) satisfies $G X=K$, independent of the operator theory result based on the commutant lifting theorem. To illustrate this we give a direct proof of the identity $G U=K V$, using the realizations of $U$ and $V$ given by (5.14) and (5.13), respectively. The direct proof requires a number of non-trivial identities which are given by the following lemma.

Lemma 5.4. Let $\left[\begin{array}{ll}G & K\end{array}\right]$ be given by the observable stable realization in (1.3), and assume that items $(i)$ and $($ ii $)$ in Theorem 1.1 are satisfied. Define $\Omega_{0}=$ $I+\left(P_{2}-P_{1}\right) Q$. Then the following identities hold:

$$
\begin{aligned}
& B_{1} C_{1}-B_{2} C_{2}=A \Omega_{0}-\Omega_{0} A_{0}, \\
& D_{1} C_{1}-D_{2} C_{2}=C \Omega_{0}, \\
& B_{1} D_{U}-B_{2} D_{V}=-\Omega_{0} B_{0}, \\
& D_{1} D_{U}-D_{2} D_{V}=0 .
\end{aligned}
$$

Here $C_{1}$ and $C_{2}$ are given by (1.13), and the matrices $A_{0}$ and $C_{0}$ are given by (5.1).

For the moment let us assume that the above identities are proved, and let us consider $G(z) U(z)-K(z) V(z)$. Using the realizations in (5.6), (5.14), and (5.13), we see that

$$
\begin{aligned}
G(z) U(z)- & K(z) V(z)=\left(D_{1} D_{U}-D_{2} D_{V}\right)+ \\
& +z C\left(I_{n}-z A\right)^{-1}\left(B_{1} D_{U}-B_{2} D_{V}\right) \\
& +z\left(D_{1} C_{1}-D_{2} C_{2}\right)\left(I_{n}-z A_{0}\right)^{-1} B_{0} \\
& +z C\left(I_{n}-z A\right)^{-1}\left(z B_{1} C_{1}-z B_{2} C_{2}\right)\left(I_{n}-z A_{0}\right)^{-1} B_{0} .
\end{aligned}
$$

Now using the identity (5.15) we see that

$$
\begin{aligned}
\left(I_{n}-z A\right)^{-1} & \left(z B_{1} C_{1}-z B_{2} C_{2}\right)\left(I_{n}-z A_{0}\right)^{-1}= \\
& =\left(I_{n}-z A\right)^{-1}\left(z A \Omega_{0}-z \Omega_{0} A_{0}\right)\left(I_{n}-z A_{0}\right)^{-1} \\
& =\left(I_{n}-z A\right)^{-1}\left(\Omega_{0}\left(I_{n}-z A_{0}\right)-\left(I_{n}-z A\right) \Omega_{0}\right)\left(I_{n}-z A_{0}\right)^{-1} \\
& =\left(I_{n}-z A\right)^{-1} \Omega_{0}-\Omega_{0}\left(I_{n}-z A_{0}\right)^{-1} .
\end{aligned}
$$

It follows that

$$
\begin{aligned}
G(z) U(z)- & K(z) V(z)=\left(D_{1} D_{U}-D_{2} D_{V}\right)+ \\
& +z C\left(I_{n}-z A\right)^{-1}\left(B_{1} D_{U}-B_{2} D_{V}+\Omega_{0} B_{0}\right) \\
& +z\left(D_{1} C_{1}-D_{2} C_{2}-C \Omega_{0}\right)\left(I_{n}-z A_{0}\right)^{-1} B_{0} .
\end{aligned}
$$

The identities (5.18), (5.17), and (5.16) then show that $G(z) U(z)-K(z) V(z)$ is identically equal to zero, that is, $G U=K V$. 
Proof of Lemma 5.4. In the sequel we shall use the following two identities

$$
Q-A^{*} Q A=C_{0}^{*} \Delta C_{0} \text { and } Q-A^{*} Q A_{0}=C^{*} C_{0} .
$$

These identities follow by using the definition of $A_{0}$ and $C_{0}$ together with the fact that $Q$ is a hermitian matrix satisfying (1.10).

Proof of (5.15). Using $C_{j}=D_{j}^{*} C_{0}+B_{j}^{*} Q A_{0}$ for $j=1,2$ and the second Stein equation in (5.19), we have

$$
\begin{aligned}
B_{j} C_{j} & \left.=B_{j} D^{*} C_{0}+B_{j} B_{j}^{*} Q A_{0}=B_{j} D_{j}^{*} C_{0}+\left(P_{j}-A P_{j} A^{*}\right) Q A_{0} \quad \text { [by [1.9] }\right] \\
& =B_{j} D_{j}^{*} C_{0}+P_{j} Q A_{0}-A P_{j} A^{*} Q A_{0} \\
& =B_{j} D_{j}^{*} C_{0}+P_{j} Q A_{0}-A P_{j}\left(Q-C^{*} C_{0}\right) \quad[\text { by the second part of [5.19)] } \\
& =\left(B_{j} D_{j}^{*}+A P_{j} C^{*}\right) C_{0}+P_{j} Q A_{0}-A P_{j} Q \quad(j=1,2) .
\end{aligned}
$$

Taking differences we obtain:

$$
\begin{aligned}
B_{1} C_{1}-B_{2} C_{2}= & \left(B_{1} D_{1}^{*}-B_{2} D_{2}^{*}+A\left(P_{1}-P_{2}\right) C^{*}\right) C_{0}+ \\
& \quad+\left(P_{1}-P_{2}\right) Q A_{0}-A\left(P_{1}-P_{2}\right) Q \\
= & \Gamma C_{0}+\left(P_{1}-P_{2}\right) Q A_{0}-A\left(P_{1}-P_{2}\right) Q \quad[\text { by [1.8)] } \\
= & A-A_{0}+\left(P_{1}-P_{2}\right) Q A_{0}-A\left(P_{1}-P_{2}\right) Q \quad[\text { by [5.1)] }] \\
= & A\left(I+\left(P_{2}-P_{1}\right) Q\right)-\left(I+\left(P_{2}-P_{1}\right) Q\right) A_{0}=A \Omega_{0}-\Omega_{0} A_{0} .
\end{aligned}
$$

Hence (5.15) holds.

Proof of (5.16). Again using $C_{j}=D_{j}^{*} C_{0}+B_{j}^{*} Q A_{0}$ for $j=1,2$ we have

$$
\begin{aligned}
D_{1} C_{1}-D_{2} C_{2}= & \left(D_{1} D_{1}^{*}-D_{2} D_{2}^{*}\right) C_{0}+\left(D_{1} B_{1}^{*}-D_{2} B_{2}^{*}\right) Q A_{0} \\
= & \left(D_{1} D_{1}^{*}-D_{2} D_{2}^{*}\right) C_{0}+\left(\Gamma^{*}-C\left(P_{1}-P_{2}\right) A^{*}\right) Q A_{0} \quad[\text { by (1.8) }] \\
= & \left(D_{1} D_{1}^{*}-D_{2} D_{2}^{*}\right) C_{0}+\Gamma^{*} Q A_{0}-C\left(P_{1}-P_{2}\right) A^{*} Q A_{0} \\
= & \left(D_{1} D_{1}^{*}-D_{2} D_{2}^{*}\right) C_{0}+\Gamma^{*} Q\left(A-\Gamma C_{0}\right)+\quad[\text { by (5.1)] }] \\
& \quad-C\left(P_{1}-P_{2}\right)\left(Q-C^{*} C_{0}\right) \quad[\text { by (5.19)] }] \\
= & \left(D_{1} D_{1}^{*}-D_{2} D_{2}^{*}+C\left(P_{1}-P_{2}\right) C^{*}\right) C_{0}+ \\
& \quad+\Gamma^{*} Q A-\Gamma^{*} Q \Gamma C_{0}-C\left(P_{1}-P_{2}\right) Q \\
= & \left(R_{0}-\Gamma^{*} Q \Gamma\right) C_{0}+\Gamma^{*} Q A-C\left(P_{1}-P_{2}\right) Q \quad[\text { by [1.7 }] \\
= & C-\Gamma^{*} Q A+\Gamma^{*} Q A-C\left(P_{1}-P_{2}\right) Q \quad[\text { by (5.1)] }] \\
= & C\left(I+\left(P_{2}-P_{1}\right) Q\right)=C \Omega_{0} .
\end{aligned}
$$

Thus (5.16) holds.

Proof of (5.17). To establish (5.17) we use that $B_{0}=B_{2}-\Gamma D_{0}+A \Omega C_{2}^{*}$. This identity follows from

$$
\begin{aligned}
B_{0} & =B_{2}-\Gamma \Delta^{-1}\left(D_{2}-\Gamma^{*} Q B_{2}\right)+A_{0} \Omega C_{2}^{*} \\
& =B_{2}-\Gamma \Delta^{-1}\left(D_{2}-\Gamma^{*} Q B_{2}\right)+\left(A-\Gamma C_{0}\right) \Omega C_{2}^{*} \\
& =B_{2}-\Gamma D_{0}+A \Omega C_{2}^{*} .
\end{aligned}
$$


Using (1.8) we see that

$$
\begin{aligned}
& B_{1} D_{U}-B_{2} D_{V}+\Omega_{0} B_{0}= \\
& =-B_{2}+\left(B_{1} D_{1}^{*}-B_{2} D_{2}^{*}\right) D_{0}+\left(B_{1} B_{1}^{*}-B_{2} B_{2}^{*}\right) Q B_{0}+\Omega_{0} B_{0} \\
& =-B_{2}+\left(\Gamma+A\left(P_{2}-P_{1}\right) C^{*}\right) D_{0}+\left(B_{1} B_{1}^{*}-B_{2} B_{2}^{*}\right) Q B_{0}+\Omega_{0} B_{0} \\
& =-B_{2}+\Gamma D_{0}+A\left(P_{2}-P_{1}\right) C^{*} D_{0}+\left(B_{1} B_{1}^{*}-B_{2} B_{2}^{*}\right) Q B_{0}+ \\
& \quad+\left(I+\left(P_{2}-P_{1}\right) Q\right) B_{0} \\
& \quad A \Omega C_{2}^{*}+A\left(P_{2}-P_{1}\right) C^{*} D_{0}+\left(B_{1} B_{1}^{*}-B_{2} B_{2}^{*}\right) Q B_{0}+\left(P_{2}-P_{1}\right) Q B_{0},
\end{aligned}
$$

using (5.20) in the last identity. Next we use (1.9). This yields

$$
\begin{aligned}
& B_{1} D_{U}-B_{2} D_{V}+\left(I+\left(P_{2}-P_{1}\right) Q\right) B_{0}= \\
& =A \Omega C_{2}^{*}+A\left(P_{2}-P_{1}\right) C^{*} D_{0}+ \\
& \quad+\left(\left(P_{1}-P_{2}\right)-A\left(P_{1}-P_{2}\right) A^{*}\right) Q B_{0}+\left(P_{2}-P_{1}\right) Q B_{0} \\
& =A \Omega C_{2}^{*}+A\left(P_{2}-P_{1}\right)\left(C^{*} D_{0}+A^{*} Q B_{0}\right) .
\end{aligned}
$$

We proceed by computing $C^{*} D_{0}+A^{*} Q B_{0}$. We have

$$
\begin{aligned}
C^{*} D_{0}+A^{*} Q B_{0} & =C^{*} D_{0}+A^{*} Q B_{2}-A^{*} Q \Gamma D_{0}+A^{*} Q A \Omega C_{2}^{*} \quad[\text { by (15.20) }] \\
& =\left(C^{*}-A^{*} Q \Gamma\right) D_{0}+A^{*} Q B_{2}+A^{*} Q A \Omega C_{2}^{*} \\
& =C_{0}^{*} \Delta D_{0}+A^{*} Q B_{2}+A^{*} Q A \Omega C_{2}^{*} .
\end{aligned}
$$

Now observe that $C_{0}^{*} \Delta D_{0}=C_{0}^{*}\left(D_{2}-\Gamma^{*} Q B_{2}\right)+C_{0}^{*} \Delta C_{0} \Omega C_{2}^{*}$. Using this together with the first identity in (5.19) we obtain

$$
\begin{aligned}
C^{*} D_{0}+A^{*} Q B_{0} & =C_{0}^{*} D_{2}+\left(A^{*}-C_{0}^{*} \Gamma^{*}\right) Q B_{2}+A^{*} Q A \Omega C_{2}^{*}+C_{0}^{*} \Delta C_{0} \Omega C_{2}^{*} \\
& =C_{0}^{*} D_{2}+A_{0}^{*} Q B_{2}+Q \Omega C_{2}^{*} \\
& =C_{2}^{*}+Q \Omega C_{2}^{*}=\left(I_{n}+Q \Omega\right) C_{2}^{*} .
\end{aligned}
$$

Summarizing we have:

$$
B_{1} D_{U}-B_{2} D_{V}+\Omega_{0} B_{0}=A\left(\Omega+\left(P_{2}-P_{1}\right)+\left(P_{2}-P_{1}\right) Q \Omega\right) C_{2}^{*}
$$

Now write $\Omega$ in (1.11) as $\Omega=-N(I+Q N)^{-1}$, where $N=P_{2}-P_{1}$. We see that

$$
\begin{aligned}
\Omega+\left(P_{2}\right. & \left.-P_{1}\right)+\left(P_{2}-P_{1}\right) Q \Omega= \\
& =-N(I+Q N)^{-1}+N+N Q\left(-N(I+Q N)^{-1}\right) \\
& =-N(I+Q N)^{-1}+N-N(Q N+I-I)(I+Q N)^{-1} \\
& =-N(I+Q N)^{-1}+N(I+Q N)^{-1}=0 .
\end{aligned}
$$

Therefore we obtain (5.17). 
Proof of (5.18). To establish (5.18) notice that

$$
\begin{aligned}
D_{1} D_{U} & -D_{2} D_{V}=D_{1}\left(D_{1}^{*} D_{0}+B_{1}^{*} Q B_{0}\right)-D_{2}-D_{2}\left(D_{2}^{*} D_{0}+B_{2}^{*} Q B_{0}\right) \\
= & \left(D_{1} D_{1}^{*}-D_{2} D_{2}^{*}\right) D_{0}+\left(D_{1} B_{1}^{*}-D_{2} B_{2}^{*}\right) Q B_{0}-D_{2} \\
= & \left(R_{0}+C\left(P_{2}-P_{1}\right) C^{*}\right) D_{0}-D_{2}+ \\
& +\left(\Gamma^{*}+C\left(P_{2}-P_{1}\right) A^{*}\right)\left(Q B_{2}-Q \Gamma D_{0}+Q A \Omega C_{2}^{*}\right) \quad[\text { use (1.8)] and (15.20)] }] \\
= & \left(R_{0}-\Gamma^{*} Q \Gamma+C\left(P_{2}-P_{1}\right)\left(C^{*}-A^{*} Q \Gamma\right)\right) D_{0}+ \\
& +\left(\Gamma^{*} Q+C\left(P_{2}-P_{1}\right) A^{*} Q\right)\left(B_{2}+A \Omega C_{2}^{*}\right)-D_{2} \\
= & \left(\Delta+C\left(P_{2}-P_{1}\right) C_{0}^{*} \Delta\right)\left(\Delta^{-1}\left(D_{2}-\Gamma^{*} Q B_{2}\right)+C_{0} \Omega C_{2}^{*}\right)+ \\
& +\left(\Gamma^{*} Q+C\left(P_{2}-P_{1}\right) A^{*} Q\right)\left(B_{2}+A \Omega C_{2}^{*}\right)-D_{2} \\
= & C\left(P_{2}-P_{1}\right) C_{0}^{*} D_{2}+C\left(P_{2}-P_{1}\right)\left(A^{*}-C_{0}^{*} \Gamma^{*}\right) Q B_{2}+ \\
& +\left(\Delta C_{0}+C\left(P_{2}-P_{1}\right) C_{0}^{*} \Delta C_{0}+\Gamma^{*} Q A+C\left(P_{2}-P_{1}\right) A^{*} Q A\right) \Omega C_{2}^{*} \\
= & C\left(P_{2}-P_{1}\right) C_{2}^{*}+ \\
& +\left(\Delta C_{0}+C\left(P_{2}-P_{1}\right) Q+\Gamma^{*} Q A\right) \Omega C_{2}^{*} \quad[\text { by the first part of ([5.19)}] \\
= & C\left(P_{2}-P_{1}+\Omega+\left(P_{2}-P_{1}\right) Q \Omega\right) C_{2}^{*}=0,[\text { because of [(5.21) }] .
\end{aligned}
$$

Therefore (5.18) holds.

As the identities in Lemma 5.4 show the matrices appearing in Theorems 1.1 and 1.2 have a lot of structure. As a further illustration of this fact we mention without proof the following identity:

$$
C_{1}^{*} C_{1}-C_{2}^{*} C_{2}=\left(Q+Q\left(P_{2}-P_{1}\right) Q\right)-A_{0}^{*}\left(Q+Q\left(P_{2}-P_{1}\right) Q\right) A_{0}
$$

See also (5.4).

\section{REFERENCES}

[1] W.F. Arnold III and A.J. Laub, Generalized eigenproblem algorithms and software for algebraic riccati equations, Proc. IEEE 72 (1984), 1746-1754.

[2] M. Bakony and H.J. Woerdeman, Matrix completions, moments, and sums of Hermitian squares, Princeton Univ. Press, Princeton, 2011

[3] J.A. Ball and T.T. Trent, Unitary colligations, reproducing kernel Hilbert spaces, and Nevanlinna-Pick interpolation in several variables, J. Funct. Anal. 157 (1998), 1-61.

[4] H. Bart, I. Gohberg, M.A. Kaashoek, and A.C.M. Ran, Factorization of matrix and operator functions: the state space method, Oper. Theory Adv. Appl. 178, Birkhäuser Verlag, Basel, 2008.

[5] H. Bart, I. Gohberg, M.A. Kaashoek, and A.C.M. Ran, A state space approach to canonical factorization with applications, Oper. Theory Adv. Appl. 200, Birkhäuser Verlag, Basel, 2010.

[6] L. Carlson, Interpolation by bounded analytic functions and the corona problem, Ann. Math. 76 (1962), 547-559.

[7] M.J. Corless and A.E. Frazho, Linear sytems and control, Marcel Dekker, Inc., New York, 2003.

[8] C. Foias and A.E. Frazho, The Commutant Lifting Approach to Interpolation Problems, Oper. Theory Adv. Appl. 44, Birkhäuser-Verlag, Basel, 1990. 
[9] C. Foias, A.E. Frazho, I. Gohberg, and M.A. Kaashoek, Metric constrained interpolation, commutant lifting and systems, Oper. Theory Adv. Appl. 100, Birkhäuser Verlag, Basel, 1998.

[10] A.E. Frazho and W. Bosri, An operator perspective on signals and systems, Oper. Theory Adv. Appl. 204, Birkhäuser Verlag, Basel, 2010.

[11] A.E. Frazho, S. ter Horst, and M.A. Kaashoek, State space formulas for stable rational matrix solutions of a Leech problem, Indag. Math. (N.S.) 25 (2014), 250-274.

[12] A.E. Frazho, M.A. Kaashoek, and A.C.M. Ran, The non-symmetric discrete algebraic Riccati equation and canonical factorization of rational matrix functions on the unit circle, Integr. Equ. Oper. Theory 66 (2010), 215-229.

[13] A.E. Frazho, M.A. Kaashoek, and A.C.M. Ran, Right invertible multiplication operators and stable rational matrix solutions to an associate Bezout equation, I: the least squares solution, Integr. Equ. Oper. Theory 70 (2011), 395-418.

[14] A.E. Frazho, M.A. Kaashoek, and A.C.M. Ran, Right invertible multiplication operators and stable rational matrix solutions to an associate Bezout equation, II: Description of all solutions, Operators and Matrices 6 (2012), 833-857.

[15] P. Fuhrmann, On the corona theorem and its applications to spectral problems in Hilbert space, Trans. Amer. Math. Soc. 132 (1968), 55-66.

[16] S. ter Horst, Rational matrix solutions to the Leech equation: The Ball-Trent approach revisited, J. Math. Anal. Appl. 408 (2013), 335-344.

[17] M.A. Kaashoek and J. Rovnyak, On the preceding paper by R. B. Leech, Integr. Equ. Oper. Theory 78 (2014), 75-77.

[18] R.B. Leech, Factorization of analytic functions and operator inequalities, Integr. Equ. Oper. Theory $\mathbf{7 8}$ (2014), 71-73.

[19] M. Rosenblum and J. Rovnyak, Hardy classes and operator theory, Oxford Mathematical Monographs, Oxford Science Publications, The Clarendon Press, Oxford University Press, New York, 1985.

[20] T.T. Trent, A constructive proof of the Leech theorem for rational matrix functions, Integr. Equ. Oper. Theory 75 (2013), 39-48.

[21] S. Wahls, and H. Boche, Lower bounds on the infima in some $\mathcal{H}_{\infty}$ optimization problems, IEEE transactions on automatic control 57 (2012), 788-793.

[22] S. Wahls, H. Boche, and V. Pohl, Zero-forcing precoding for frequency selective MIMO channels with $H^{\infty}$ criterion and causality constraint, Signal Processing 89 (2009), 17541761 .

Department of Aeronautics and Astronautics, Purdue University, West Lafayette, IN 47907, USA

E-mail address: frazho@ecn.purdue.edu

Unit for BMi, North-West University, Private Bag X6001-209, Potchefstroom 2520, South AFrica

E-mail address: sanne.terhorst@nwu.ac.za

Department of Mathematics, VU University Amsterdam, De Boelelaan 1081A, 1081 HV Amsterdam, The Netherlands

E-mail address: m.a.kaashoek@vu.nl 